\title{
Strong and Weak Virtual Synchrony in Horus*
}

\author{
Roy Friedman Robbert van Renesse \\ Department of Computer Science \\ Cornell University \\ Ithaca, NY 14853.
}

August 24, 1995

\begin{abstract}
A formal definition of strong virtual synchrony, capturing the semantics of virtual synchrony as implemented in Horus, is presented. This definition has the nice property that every message is delivered within the view in which it was sent. However, it is shown that in order to implement strong virtual synchrony, the application program has to block messages during view changes.

An alternative definition, called weak virtual synchrony, which can be implemented without blocking messages, is then presented. This definition still guarantees that messages will be delivered within the view in which they were sent, only that it uses a slightly weaker notion of what the view in which a message was sent is. An implementation of weak virtual synchrony that does not block messages during view changes is developed, and it is shown how to use a system that provides weak virtual synchrony even when strong virtual synchrony is actually needed.

To capture additional ordering requirements, the definition of ordered virtual synchrony is presented. Finally, it is discussed how to extend the definitions in order to cope with the fact that a process can become a member of more than one group.
\end{abstract}

\footnotetext{
*This work was supported by ARPA/ONR grant N00014-92-J-1866
} 


\section{Introduction}

Virtual synchrony is a convenient paradigm for developing distributed applications in asynchronous systems in which processes may crash, messages may get lost, and the communication network may get partitioned, since it simulates a reliable delivery fail-stop model to the application. That is, virtual synchrony creates an illusion to the application that it runs in an environment in which crashed processes are always detected, and if a certain process is suspected of being crashed, then this process has really crashed. This is done by presenting processes with views, which consists of the set of currently reachable and operational processes. The system then guarantees that between every two consecutive views $v_{1}$ and $v_{2}$, no message that was sent from a process not in $v_{1}$ can be delivered and that all processes that appear in both $v_{1}$ and $v_{2}$ have to see the same set of messages.

In particular, the use of virtually synchronous communication systems greatly simplifies the task of developing replicated services: It it possible to send a message to the entire set of processes, and the system would ensure that all live replicas will receive a copy of the message. Moreover, if one of the replicas would become faulty, other replicas will learn about it by receiving a new view which does not include the crashed replica, and one of the live replicas would take over the job of the crashed replica. Also, in the event of a network partition, each partition will receive a view which includes only the processes that belong to that partition. Later, when the communication links are fixed, a specific join request would allow the two partitions to negotiate a new state. Finally, after the combined state of the two partitions has been resolved, a new view which consists of all processes would be generated for all participating processes.

During the course of years, several systems that support a virtually synchronous communication paradigm have been developed $[1,2,3,5,25]$. These systems usually perform quite well in terms of average message latencies, but exhibit harsh slow downs during view changes, making them impractical for applications that require timed response. In this paper, we report a formal and practical study of the membership layer of Horus [24], i.e., the layer which is responsible for implementing virtual synchrony, aimed at improving this situation. That is, we would like to come up with a membership layer that will be very efficient in the normal case, and will continue to perform well during view changes.

We start by presenting a formal definition of strong virtual synchrony, capturing the semantics of virtual synchrony as it is implemented by Horus. This is the first time that a formal definition of the membership layer of Horus is given. The definition of strong virtual synchrony includes a requirement that a message is delivered only within the view in which it was sent. The only other definition to include this requirement is the definition of extended virtual synchrony, which is supported by the Transis project [1,2] and the Totem project [3], but is not supported by other definitions of virtual synchrony, including the definition used by ISIS $[5,7]$.

We believe that this additional requirement is important for many applications, since it allows for immediate local delivery of messages, without any further computations or book- 
keeping. In other systems that do not have this guarantee $[4,5,7,9]$, local deliveries must be delayed until the system can figure out in what view the message is supposed to be delivered. Also, the fact that messages are delivered within the view in which they were sent can reduce the amount of information that needs to be sent with each message, and simplifies the computation which is needed in order to handle each message. For example, the CAUSAL and ORDER layers of Horus implements causal [15] and uniform [13, 20] delivery of messages by using vector timestamps. The fact that the view of the sender when a message is sent is identical to the views of the receivers when the message is delivered, allows the sender to send only a local vector timestamp without any additional context information. The recipients of the message can safely assume that the $i$ th entry of the vector they received corresponds to the $i$ th member in their view.

The current implementation of the membership layer of Horus blocks messages during view changes. In this paper, we show that this blocking is necessary in order to support strong virtual synchrony. Specifically, we show a lower bound, stating that in every implementation of strong virtual synchrony, there exists a time $d$ such that messages cannot be sent at least $d$ units of time before a view change. Here, $d$ is the message delay of the underlying system (layers), including the network itself.

The lower bound that we show depends on the requirement that messages are always delivered within the view in which they are sent. On the other hand, in other systems that do not support this requirement, local deliveries must incur some delays and possibly additional bookkeeping, while some applications become more complex due to the need to add more context information to every message. The immediate question that comes up is if this is a real trade-off, or can we enjoy the benefits of both worlds? In this paper we give a partial answer to this question by presenting the definition of weak virtual synchrony, which allows for immediate local deliveries, but does not require blocking messages during view changes and adds only little additional header information to messages. According to the definition of weak virtual synchrony, during view changes, processes are supplied with a temporary suggested view. Processes can send messages in a suggested view, and are guaranteed that this message will be delivered within the next real view, and that the next real view will be an ordered subset of the suggested view. We explain below the usefulness of these guarantees in reducing the context information that needs to be sent when compared with other definitions that allow to continue sending messages during view changes.

We have developed a protocol that provides weak virtual synchrony and does not block messages during view changes. In the actual implementation of this protocol in Horus, during views and suggested views, processes receive a membership list and an indication which of the processes that appear in the membership list have failed. However, due to the guarantees of suggested views, the membership list which is passed to the application is only changed in the first suggested view event after a real view event and in real view events. In other suggested view events, the membership list remains the same, and only the indication about which processes have failed may change. Hence, for most messages, the membership list is the same both when they are sent and when they are received. The only two cases where this may 
not hold are the following: $(a)$ a message that was sent between a suggested view event and the next view event, but was received after the view event, and $(b)$ a message that was sent when a view is installed, but is received after a following suggested view event. For these messages, the application can maintain a translation table, between the ranks of the members in the old list and their ranks in the current list. We have used this technique in order to extend many existing layers of Horus to work with weak virtual synchrony, which turned out to be a very simple task.

Of course, the same thing can be done with other definitions of virtual synchrony. However, with weak virtual synchrony, the application needs to maintain at most two translation tables at a time. In order to do the same with other definitions of virtual synchrony that allow to send messages during view changes, the application may need to maintain an arbitrary large number of translation tables simultaneously. And, of course, other definitions of virtual synchrony that allow to send messages during view changes do not allow for immediate local deliveries.

We have compared the latency of messages sent during view changes in both the implementation of strong virtual synchrony and the implementation of weak virtual synchrony with the latency of messages sent during normal operation. Our measurements indicate that messages sent during view changes in the weakly virtually synchronous implementation are slower than regular messages only by a small constant factor that does not depend on the load of the system. On the other hand, messages that were sent during view changes in the strongly virtually synchronous implementation were significantly slower than those sent in the weakly virtually synchronous implementation, and this gap becomes larger as the load on the system increases.

Our definitions of weak and strong virtual synchrony do not impose any ordering restrictions on messages sent within the same view. However, in many distributed applications, it is useful to have at least some of the messages delivered either in a total order or in a causal order. To capture these ordering requirements, we add the definition of ordered virtual synchrony and a short discussion about how these requirements are implemented in Horus.

Finally, we discuss how to extend our definitions so they can cope with the fact that a process may become a member of more than one group. We introduce the notion of failure domains, which means that if a process becomes a suspect in one group, it must be declared suspect in all the groups in which it is a member. We suggest a formal definition for failure domains, although this definition is slightly weaker than the semantics provided by Horus in the case that a process can be suspected of being faulty more than once.

The rest of this paper is organized as follows: Related work is discussed in Section 2. We present the formal model in Section 3. The definition of strong virtual synchrony and the lower bound on its implementations is presented in Section 4. The definition of weak virtual synchrony is presented in Section 5 while the protocol for implementing weak virtual synchrony together with the latency measurements are given in Section 6. In Section 7 we present the definition of ordered virtual synchrony. Multiple groups and failure domains are discussed in Section 8 and we conclude with a discussion in Section 9 . 


\section{Related Work}

The notion of virtual synchrony was first introduced by Ken Birman in the ISIS project $[5,8,7]$. However, this definition of virtual synchrony in ISIS is somewhat different than our definitions, as it does not provide any guarantee on the view in which a message will be delivered. Also, the definition of virtual synchrony in ISIS requires the existence of a primary partition. Having a primary partition is important for applications in order to acquire locks, or to perform any kind of operations which require coherent behavior, e.g., directing an airplane to a certain area in the sky. Our definitions allow keeping track of a primary partition, given some reasonable rules for deciding if a certain partition is primary or not. An example of such a rule can be the majority of processes within a fixed group of processes. On the other hand, our definition does not require to have only one primary partition at all times, so applications that can safely make progress even in the absence of a primary partition, will be allowed do so. As in our definitions, virtual synchrony in ISIS does not require uniformity $[20,13]$ of regular messages (also called safe delivery in [3]), i.e., that if a message is received by any process, faulty or non-faulty, then every non-faulty process must receive it too. The decision not to support uniformity of messages is motivated by the high cost associated with providing this guarantee. However, in Horus, uniformity of messages can be added by using the ORDER layer [24].

Throughout the years, several other definitions of virtual synchrony were introduced, at different levels of formality, and several algorithms for implementing them were also developed. These works include projects like Transis [1, 2], Totem [3], Relacs [4], and Newtop [12], as well as several other papers like $[9,10,23] .{ }^{1}$ Some of these works, e.g., $[1,2,3,10,23]$, include a variant of virtual synchrony which requires uniformity of messages, but others, e.g., [4, 9, 12], do not. The only other definition that include the requirement that a message is always delivered within the view in which it was sent is the definition of extended virtual synchrony which is supported by Transis [1,2] and Totem [3]. However, except for the Relacs protocol [4], and the Newtop protocol [12], all of the protocols for providing virtual synchrony developed in these works stop sending messages during the installation of new views, sometimes called the reformation phase. This blocking of messages might be inherent in the protocols themselves, but is not required by the semantics that these protocols provide. (We want to make clear that there is a difference between what the protocol does internally, and what the semantics that this protocols presents to the application is. In particular, it is possible that if some of these protocols would have added a flush event to notify the application that a configuration change is taking place, the resulting semantics would have been similar to strong virtual synchrony. However, the fact is that these protocols do not inform the application when they block its messages, so the application cannot take advantage of this.)

The definition of extended virtual synchrony [3] includes the notion of transitional view, which must be delivered before a real view can be installed. The difference between a suggested view and a transitional view is that a suggested view is a superset of the next real view, and

\footnotetext{
${ }^{1}$ The work of Chang and Maxemchuk [10] does not use the term virtual synchrony, but defines a very similar semantics.
} 
is used to allow processes to continue sending messages during view changes. In contrast, a transitional view consists of the processes that appear in the intersection of the previous and next real views, and is used to notify the application that all messages sent in the previous real view are now stable w.r.t. to all the processes that appeared in that view.

One major difference between Horus and most other systems which provide virtual synchrony is that Horus is a layered system, in which different concerns are decoupled, and each concern is implemented in a different layer. The definitions of strong and weak virtual synchrony developed in this paper follow this guideline. Hence, issues like uniformity (safe delivery), causal ordering, and atomic delivery of messages are not part of these definitions, and are not implemented in the membership layer of Horus. However, there are separate layers which can add any of these requirements to the overall semantics, in order to support applications that need a stronger semantics.

We do not claim that virtual synchrony is necessary for all types of applications. For instance, applications which are very asynchronous may prefer a model in which a message that was sent to a crashed process will be delivered to this process when it is re-booted. Examples of systems that provide such a model include GTS [17], the ISIS wide-area facility [18], and the Long-Term layer of Horus. (In fact, the Long-Term layer of Horus runs on top of the membership layer, and uses the regular membership layer to propagate messages to the set of currently active processors.)

The Psync protocol [21] provides causal delivery of messages among a fixed set of processes. Psync does not support additional properties like total ordering or dynamic membership changes, but provides hooks that enable higher level applications to do so, as done in Consul [19].

Other related work is transaction-based technology, for example, such as used in the Harp file system [16]. However, because these systems use only totally ordered transactions, their performance is much worse than what can be achieved with Horus, and there are many applications, e.g., replicated servers and mission control systems, that do not need the semantics of atomic transactions. A more detailed discussion of these issues appears in $[6,14,26]$.

Since the membership layer of Horus is implemented on top of layers which do not provide virtual synchrony, any potential application of Horus can also be developed directly with pointto-point communication. However, we believe that, in general, developing a reliable distributed application on top of a virtually synchronous system like Horus is simpler than without such a layer. This is because virtual synchrony shields the programmer from many of the bad scenarios that can otherwise happen in a distributed environment, resulting in fewer lines of code, simpler code, and a higher degree of confidence in the correctness of the implementation.

In a recent paper, Ricciardi and Birman have formalized the notion of group membership using temporal logic in [22]. However, this definition deals only with view changes, and does not refer to other messages and their ordering. 


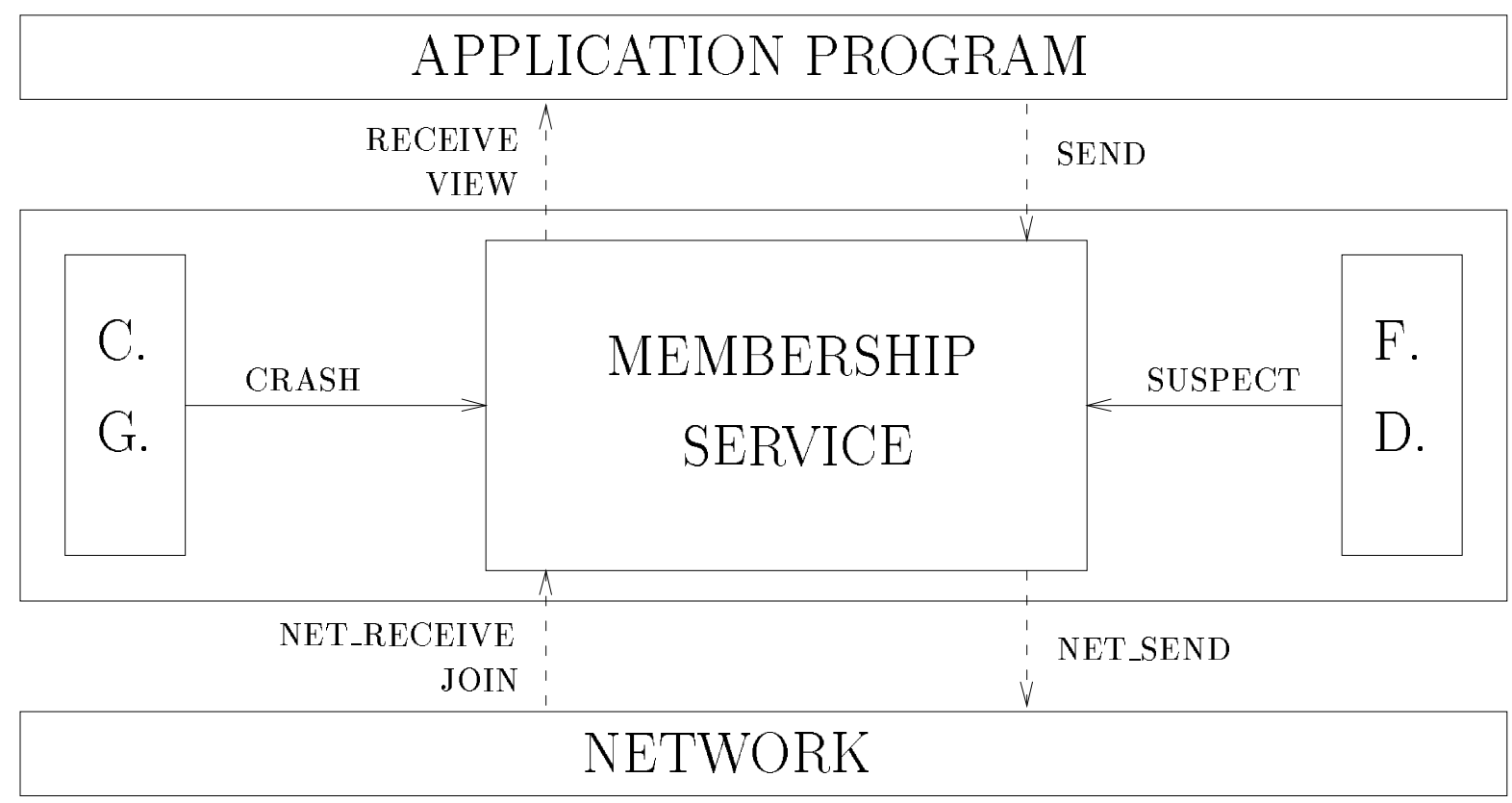

Figure 1: System illustration

\section{The Model}

We assume a finite (possibly unbounded) set of nodes $S$, connected via some interconnection network. Each node in $S$ consists of the following components: an application program, a membership service, a failure detector, and a crash generator. The membership service is an automaton, that accepts events either from the network or from the other components of the same node, do some local computation, and generates zero or more events to the network and the other components of the same node. (See illustration in Figure 1.) The membership service can accept send events from the application program, suspect events from the failure detector, crash events from the crash generator, and net_receive and join events from the network; the membership service may generate receive and view events for the application program, and net_send events for the network. In the rest of this paper, when we say process we refer to the membership service.

A history $h_{i}$, is a sequence of events that occur in a process $p_{i}$, in the order of their occurrence:

view: a view event $e v$ includes an ordered list of processes, denoted by $e v . v i e w$, and a view id number, denoted by ev.vid.

send: a send event $e v$ includes a message, denoted by ev.msg, a list of processes to whom the message should be sent, denoted by ev.target, the id of the process in which the event occurs, denoted by ev.pid, and a view id number, denoted by ev.vid. 
receive: a receive event $e v$ includes a message, denoted by $e v . m s g$, and an id number of the process that sent the message, denoted by ev.pid.

join: a join event $e v$ includes a process id, denoted by $e v$.pid.

suspect: a suspect event $e v$ includes a list of processes, denoted by ev.suspects.

crash: a crash event has no parameters.

net_send: a net_send event $e v$ includes a message to be sent, denoted by ev.msg, and a list of processes to whom the message should be sent, denoted by ev.target.

net_receive: a net_receive event $e v$ includes a message, denoted by $e v . m s g$.

We assume also that messages are unique, i.e., for every two send events $e v$ and $e v^{\prime}, e v . m s g \neq$ $e v^{\prime} . m s g$ and if $e v$ appears in some history $h_{i}$, then $e v$ does not appear in any other history $h_{j}$, $j \neq i$. (This can be implemented by timestamping the messages.) Note that since a receive event and a view event are not characterized by the process in which they occurred, the same receive or view events may occur in several histories.

Given a sequence of events $h$ and two events $e v$ and $e v^{\prime}$ in $h$, we denote the fact that $e v$ appears in $h$ before $e v^{\prime}$ by $e v \stackrel{h}{\longrightarrow} e v^{\prime}$. Similarly, given an ordered list of processes $v$ and two processes $p_{i}$ and $p_{j}$ in $v$, we denote the fact that $p_{i}$ appears in $v$ before $p_{j}$ by $p_{i} \stackrel{v}{\longrightarrow} p_{j}$.

We say that a process history $h_{i}$ is admissible if the following holds:

1. If $h_{i}$ includes a crash event $e v$, then $e v$ is the last event in $h_{i}$.

2. For every send event $e v$ in $h_{i}$, there exists a view event $e v^{\prime}$ such that $e v^{\prime} \stackrel{h_{i}}{\longrightarrow} e v$, $e v^{\prime} . v i d=e v . v i d, e v \cdot t a r g e t \subseteq e v^{\prime} . v i e w$, and there does not exist another view event $e v^{\prime \prime}$ such that $e v^{\prime} \stackrel{h_{i}}{\longrightarrow} e v^{\prime \prime} \stackrel{h_{i}}{\longrightarrow} e v$.

An execution is a collection of process histories, one for each process. We say that an execution $\sigma$ is admissible if every history $h_{i}$ of $p_{i}$ in $\sigma$ is admissible and for every net_receive event $e v$ in every history $h_{i}$ in $\sigma$, there exists a net_send event $e v^{\prime}$ in $h_{e v . p i d}$ such that $e v . m s g=$ $e v^{\prime} . m s g$ and $p_{i} \in e v^{\prime}$.target. From now on, we assume that all executions are admissible.

We assume in this paper that the system is asynchronous. That is, different processes may run at a different rate, and the delay of messages is unknown, and may vary from one message to another. For reasons of time analysis, we assume that each event $e v$ in every history $h_{i}$ is associated with some real time $\mathcal{R}_{h_{i}}(e v)$. We define $\mathcal{R}_{h_{i}}(e v)$ to be $\perp$ if $e v$ is not included in $h_{i}$. We emphasize that a process may not know the real time associated with the events in its history. Given a send event $e v$ in some history $h_{i}$ of an execution $\sigma$, and a receive event $e v^{\prime}$ in a history $h_{j}$ of $\sigma$ such that $e v . m s g=e v^{\prime} . m s g$, we define $d_{j}(e v . m s g)=\mathcal{R}_{h_{j}}\left(e v^{\prime}\right)-\mathcal{R}_{h_{i}}(e v)$; we define $d(e v \cdot m s g)$, the delay of the message, to be the maximum of $d_{j}(e v . m s g)$ over all $j$ s for 
which $e v^{\prime}$ is included in $h_{j}$, and to be $\infty$ if $e v^{\prime}$ does not exist in any history of $\sigma$. We assume that for every send event $e v, d_{j}(e v . m s g)>0$.

A protocol or an algorithm is any description of the transition function of the membership service, i.e., any description of the local computations and events that should be generated by the membership service whenever it accepts an event.

Note, that we do not explicitly model the failure detector, the application program, or the communication network. However, the behavior of these elements must conform with the other requirements of the system. In particular, the network may not generate spurious messages, and may not duplicate messages. However, whether the actual network has these characteristics, or in fact the network is totally unreliable, but there is a layer which sits between the "real" network and the interface to the membership service in order to provide these guarantees, is none of our concern in this paper. This matches the approach of Horus, in which each layer implements only its own functionality, and does not care which layers are placed below and how they are implemented, as long as these lower layers provide the right semantics.

Note also that Figure 1 illustrate the system architecture as it appears in a single node. This is why the events of the crash generator are sent to the membership service and not to the failure detector. That is, a crash event generated by the crash generator only causes the local membership service to crash by halting. (In particular, crashes do not cause the membership service to generate spurious messages).

\section{Strong Virtual Synchrony}

In this section, we define the notion of strong virtual synchrony formally, capturing the original semantics provided by the membership layer of Horus. We then show that these semantics impose long delays on messages that are about to be sent just before a view change.

The definition of strong virtual synchrony is divided into two parts. In the first part, we define the requirements from views, while the second part includes the requirements about the ordering of messages w.r.t. these views. However, before we can introduce these definitions, we need the following notion of consecutive view events in a history. Formally, we say that two

view events $e v^{\prime}$ and $e v^{\prime \prime}$ are consecutive view events in a history $h_{i}$ if $e v^{\prime} \stackrel{h_{i}}{\longrightarrow} e v^{\prime \prime}$ and there does not exists another view event $e v^{\prime \prime \prime}$ such that $h_{i}$ if $e v^{\prime} \stackrel{h_{i}}{\longrightarrow} e v^{\prime \prime \prime} \stackrel{h_{i}}{\longrightarrow} e v^{\prime \prime}$.

An intuitive explanation of the following definition is given in the text below it.

Definition 4.1 (Strong View Admissibility) An execution $\sigma$ is strongly view admissible if the following holds:

1. For every history $h_{i}$ in $\sigma$ and every view event $e v$ in $h_{i}, p_{i}$ is included in ev. 
2. If ev and $e v^{\prime}$ are two view events in $h_{i}$ such that $e v \stackrel{h_{i}}{\longrightarrow} e v^{\prime}$, then ev.vid $<e v^{\prime} . v i d$.

3. If some history $h_{i}$ includes a crash event ev and there exists another history $h_{j}, j \neq i$, that includes a view event $e v^{\prime}$ such that $p_{i} \in e v^{\prime} . v i e w$, then there exists another event $e v^{\prime \prime}$ in $h_{j}$ such that $e v^{\prime} \stackrel{h_{i}}{\longrightarrow} e v^{\prime \prime}$ and either $e v^{\prime \prime}$ is a view event and $p_{i} \notin e v^{\prime \prime}$.view or $e v^{\prime \prime}$ is a crash event.

4. If some history $h_{i}$ includes a join event ev and does not include a crash event, then $h_{i}$ includes another event $e v^{\prime}$ such that $e v \stackrel{h_{i}}{\longrightarrow} e v^{\prime}$ and either $e v^{\prime}$ is a view event such that ev.pid $\in e v^{\prime} . v i e w$ or $e v^{\prime}$ is a suspect event and ev.pid $\in e v^{\prime}$. suspects.

5. For every view event ev that appears in a history $h_{i}$ and every process $p_{j}$ such that $p_{j} \in$ ev.view, if ev is the last view event in $h_{i}$ and $h_{i}$ does not include a crash event, or if $e v^{\prime}$ is a consecutive view event in $h_{i}$ and $p_{j} \in e v . v i e w \cap e v^{\prime}$.view, then $h_{j}$ includes ev.

6. For every two consecutive view events ev and $e^{\prime}$ in a history $h_{i}$ and every process $p_{j}$, if $p_{j} \in e v . v i e w \backslash e v^{\prime} . v i e w$, then there exists a history $h_{k}$ that includes a suspect event ev" such that $p_{j} \in e v^{\prime \prime}$. suspects, $e v \stackrel{h_{k}}{\longrightarrow} e v^{\prime \prime}$ and there is no view or crash event ordered in $h_{k}$ between $e v$ and $e v^{\prime \prime}$.

The first condition in the definition of strong view admissibility requires that every process will be included in all of its local views. The second condition requires that the vid field of view events will reflect their relative order within a history. The third condition requires that every process that crashes will eventually be removed from the views of all processes (if it appeared there). The fourth condition requires that if a process wishes to join a view, it will either be added eventually, or declared suspect. The fifth condition requires that if process $p_{j}$ appears in two consecutive views of another process $p_{i}$, denote the first one of these views by $V$, then $p_{j}$ must have seen view $V$ as well. The sixth condition requires that a process can only be removed from a view if it was suspected of being faulty by a member of that view. (Note that in this case, $h_{k}$ is not necessarily the same as $h_{i}$ and therefore, $e v$ and $e v$ need not be consecutive view events in $h_{k}$.)

Before we can define strong virtual synchrony, we introduce the notion of the view event which generates the view in which a message is sent. Given a send event $e v$ and a view event $e v^{\prime}$, we say that $e v^{\prime}$ generates the view in which ev.msg is sent if ev.vid $=e v^{\prime} . v i d$.

An intuitive explanation of the definition below, appears in the text that follows.

Definition 4.2 (Strong Virtual Synchrony) An execution $\sigma$ is strongly virtually synchronous if the following holds:

1. $\sigma$ is strongly view admissible. 
2. For every history $h_{i}$ in $\sigma$ and every receive event $e v$ in $h_{i}$, if $e v^{\prime}$ is the view event that generates the view in which ev.msg is sent, then $e v^{\prime} \stackrel{h_{i}}{\longrightarrow}$ ev, and if there exists a view event $e v^{\prime \prime}$ in $h_{i}$ such that $e v^{\prime \prime}$. vid $>e v^{\prime}$. vid, then $e v \stackrel{h_{i}}{\longrightarrow} e v^{\prime \prime}$.

3. Let ev be a send event, $e v^{\prime}$ the view event in which ev.msg was generated, and $p_{i}$ a process such that $p_{i} \in$ ev.target, then if $e v^{\prime}$ is the last view event in both $h_{i}$ and $h_{e v . p i d}$ and neither $h_{i}$ nor $h_{\text {ev.pid }}$ includes a crash event, or if $e v^{\prime \prime}$ is a consecutive view event in both $h_{i}$ and $h_{\text {ev.pid, }}$, then there exists a receive event $e v^{\prime \prime \prime}$ in $h_{i}$ such that ev.msg $=e v^{\prime \prime \prime} . m s g$.

4. Let ev be a send event, $e v^{\prime}$ the corresponding receive event, ev" the view event in which ev.msg is generated, and $h_{i}$ a history that includes both $e v^{\prime}$ and $e v^{\prime \prime}$. Then the following holds: (a) If $e v^{\prime \prime \prime}$ is a consecutive view event in $h_{i}$, and $e v^{\prime} \stackrel{h_{i}}{\longrightarrow} e v^{\prime \prime \prime}$, then every history $h_{j}$, in which $e v^{\prime \prime}$ and $e v^{\prime \prime \prime}$ are consecutive view events, includes $e v^{\prime}$. (b) If $e v^{\prime \prime}$ is the last view event in $h_{i}$ and there are no crash events in $h_{i}$, then every history $h_{j}$, in which ev" is the last view event in $h_{j}$ and $h_{j}$ does not include a crash event, includes ev'.

5. For every history $h_{i}$ and two send events ev and $e v^{\prime}$ such that for some history $h_{j}$, $e v \stackrel{h_{j}}{\longrightarrow} e v^{\prime}$, the view in which ev.msg and $e v^{\prime} . m s g$ are generated is the same, and $p_{i} \in$ ev.target $\cap e v^{\prime}$.target, if $h_{i}$ includes the receive event that corresponds to $e v^{\prime}$, denoted by $e v^{\prime \prime \prime}$, then $h_{i}$ includes the receive event that corresponds to ev, denoted by $e v^{\prime \prime}$, and $e v^{\prime \prime} \stackrel{h_{i}}{\longrightarrow} e v^{\prime \prime \prime}$.

By the first condition in the definition of strong virtual synchrony, a strongly virtually synchronous execution must also be a strongly view admissible execution. The second condition requires that every message must be delivered within the view in which it was sent. The third condition requires that every message sent by a process that remains in the view of other processes, is delivered by these processes. This requirements guarantees reliable delivery of messages. The fourth condition requires that all "surviving" members of a view agree on the set of messages delivered within this view. The fifth condition requires that between two consecutive view events, Horus simulates a no-omission failures model among messages sent to the same destinations, and that these messages are delivered in fifo order.

A protocol $\mathcal{P}$ implements strong virtual synchrony if every execution generated by it is strongly virtually synchronous. For the rest of this paper, we use SVS as shorthand for strong virtual synchrony.

Note that the vid field is used in the definitions of admissible executions, view admissibility, and virtual synchrony only for bookkeeping and these definitions could have been written without it. However, without the vid field these definitions would have been more complex and formal reasoning about them would have been even more difficult. Also, currently there is no starting event in the definition of admissible executions. This may contradict the intuitive thought that an application program needs to do something active in order to become a member of a group. To overcome this concern, we can introduce a new event, start, that the 
membership service may accept from the application program, and require that this event will be the first event in every admissible history. We have decided not to add this event to the formal definition of admissible executions presented in this paper, since it is not required for any of the other results presented in this paper. On the other hand, we believe that the current definitions allow to develop self stabilizing protocols [11], and by adding a starting event we would have prohibited such protocols.

The following lemma shows that in every implementation of strong virtual synchrony, processes have to stop sending messages at least $d$ time before a new view is installed, where $d$ is the delay of the underlying layers (including the delay of the network itself).

Lemma 4.1 (Lower Bound for SVS) In every SVS implementation, no send event can be generated by a process $p_{i}$ at least d time before a view event is generated by any process that appears in the target field of this send event.

Proof: Assume, by way of contradiction, that there exists an SVS implementation $\mathcal{E}$ in which a send event can be generated $d^{\prime}<d$ time before a view event. Consider the following execution $\sigma$ of $\mathcal{E}$ that includes two processes $p_{0}$ and $p_{1}$ that send a message to every process in their view at least every $d^{\prime}<d$ time. We assume that there exist two consecutive view events $e v$ and $e v^{\prime}$ in $h_{0}$ and $h_{1} ; e v$ occurs at real time $t_{0}$ in $h_{0}$ and at real time $t_{1}$ in $h_{1} ; e v^{\prime}$ occurs at real time $t_{0}^{\prime}$ in $h_{0}$ and at real time $t_{1}^{\prime}$ in $h_{1}$. In particular, this means that both $p_{0}$ and $p_{1}$ are included in ev.view and $e v^{\prime}$.view. We assume also that $t_{1}^{\prime}, t_{0}^{\prime}>t_{0}+d^{\prime}$ and $t_{1}^{\prime}, t_{0}^{\prime}>t_{1}+d^{\prime}$.

Assume, without loss of generality, that $t_{1}^{\prime}>t_{0}^{\prime}$. Hence, during the time interval $\left[t_{0}^{\prime}-d^{\prime}, t_{0}^{\prime}\right]$, there is a send event $e v^{\prime \prime}$ by $p_{1}$ such that $e v^{\prime \prime} . v i d \leq e v . v i d$ and $p_{0} \in e v^{\prime \prime}$.target. Due to the message delay, the corresponding receive event occurs in $p_{0}$ after time $t_{0}^{\prime}$. Hence, $e v^{\prime \prime}$ is ordered after $e v^{\prime}$, although $e v^{\prime} . v i d>e v^{\prime \prime} . v i d$. A contradiction to the assumption that $\mathcal{E}$ is an SVS implementation.

\section{$5 \quad$ Weak Virtual Synchrony}

In order to define weak virtual synchrony, we introduce a new type of event called suggested view. A suggested view event $e v$ includes a view id number, denoted by $e v$.vid, and an ordered list of processes, denoted by ev.sview.

A process history $h_{i}$ is weakly admissible if the following holds:

a. If $h_{i}$ includes a crash event $e v$, then $e v$ is the last event in $h_{i}$.

b. For every send event $e v$ in $h_{i}$, there exists a suggested view event $e v^{\prime}$ such that $e v^{\prime} \stackrel{h_{i}}{\longrightarrow} e v$, $e v^{\prime} . v i d=e v . v i d, e v . t a r g e t \subseteq e v^{\prime} . v i e w$, and there does not exist a suggested view event $e v^{\prime \prime}$ such that $e v^{\prime} \stackrel{h_{i}}{\longrightarrow} e v^{\prime \prime} \stackrel{h_{i}}{\longrightarrow} e v$. 
An execution $\sigma$ is weakly admissible if every history in $\sigma$ is weakly admissible and for every receive event $e v$ in every history $h_{i}$ in $\sigma$, there exists a send event $e v^{\prime}$ in $h_{e v \text {.pid }}$ such that ev.msg $=e v^{\prime} . m s g$ and $p_{i} \in e v^{\prime}$. target.

Definition 5.1 (Weak View Admissibility) An execution $\sigma$ is weakly view admissible if it is weakly admissible and the following holds:

a. $\sigma$ obeys all the conditions in the definition of a strong view admissibility.

b. In every history there exists at least one suggested view event before the first view event and between every two consecutive view events.

c. For every history $h_{i}$ and every two events ev and $e v^{\prime}$ such that $e v \stackrel{h_{i}}{\longrightarrow}$ ev and each of $e v$ and $e v^{\prime}$ is either a view event or a suggested view event, ev.vid $\leq e v^{\prime} . v i d$. In particular, if $e v^{\prime}$ is a suggested view event, then ev.vid $<e v^{\prime} . v i d$.

d. For every suggested view event ev and every view or suggested view event ev' such that $e v \stackrel{h_{i}}{\longrightarrow} e v^{\prime}$ and there does not exist another view event $e v^{\prime \prime}$ such that $e v \underset{e v^{\prime} . v i e w}{\stackrel{h_{i}}{\longrightarrow}} e v^{\prime \prime} \stackrel{h_{i}}{\longrightarrow}$ $e v^{\prime}, e v^{\prime} . v i e w \subseteq e v . v i e w$ and $e v . v i d \leq e v^{\prime} . v i d$. Moreover, if a process $p_{j} \stackrel{\text { ev } . \text { view }}{\longrightarrow} p_{k}$, then $p_{j} \stackrel{\text { ev.view }}{\longrightarrow} p_{k}$.

By requirement $(d)$ in Definition 5.1, every view or suggested view, except for the first suggested view after a view event, must be an ordered subset of the previous suggested view. In particular, this means that new processes can only join in the first suggested view that follows a view event; other suggested view events, and the view event itself, can only eliminate processes from previously suggested views.

We now slightly modify the definition of the view event that generates the view in which a message is sent, and introduce the notion of the suggested view which proposes a view to a process. Let $h_{i}$ be some history, $e v$ a send event in $h_{i}$, $e v^{\prime}$ a view event, and $e v^{\prime \prime}$ the first suggested view event such that $e v^{\prime \prime} \stackrel{h_{i}}{\longrightarrow} e v^{\prime}$ and there does not exist another view event $e v^{\prime \prime \prime}$ such that $e v^{\prime \prime} \stackrel{h_{i}}{\longrightarrow} e v^{\prime \prime \prime} \stackrel{h_{i}}{\longrightarrow} e v^{\prime}$. If $e v^{\prime \prime} . v i d \leq e v . v i d \leq e v^{\prime} . v i d$, then we say that $e v^{\prime}$ generates the view in which ev.msg is sent, while ev" proposes to $p_{i}$ the view in which ev.msg is sent. Note that by this definition, a message can be sent before the event that generates the view in which it is sent.

Definition 5.2 (Weak Virtual Synchrony) An execution $\sigma$ is weakly virtually synchronous if the following holds:

a. $\sigma$ is weakly view admissible. 
b. For every history $h_{i}$ in $\sigma$ and every receive event $e v$ in $h_{i}$, if $e v^{\prime}$ is the suggested view event that proposes to $p_{\text {ev.pid }}$ the view in which ev.msg is sent, then $\mathrm{ev}^{\prime} \stackrel{h_{i}}{\longrightarrow} e v$. Moreover, if $e v^{\prime \prime}$ is the view event that generates the view in which ev.msg is sent and there exists a view event $e v^{\prime \prime \prime}$ in $h_{i}$ such that $e v^{\prime \prime \prime} . v i d>e v^{\prime \prime} . v i d$, then $e v \stackrel{h_{i}}{\longrightarrow} e v^{\prime \prime \prime}$.

c. $\sigma$ obeys Conditions 3, 4, and 5 in the definition of a strong virtual synchrony.

Note that by requirement $(b)$ in Definition 5.2 , the suggested view event that proposes the view in which a message was sent must be seen by all the processes that receive this message. For example, consider histories $h_{i}$ and $h_{j}$ that appear in Figure 2 . In this example, $v_{2}$ proposes to $p_{i}$ the view in which $m$ is sent, while $v_{3}$ generates the view in which $m$ is sent. $p_{j}$, which receive $m$, must see $v_{2}$ before the receive event of $m$. Moreover, the receive event of $m$ can be ordered either before or after $v_{4}$, but must be ordered before $v_{5}$.

A protocol $\mathcal{P}$ implements weak virtual synchrony if every execution generated by it is weakly virtually synchronous. For the rest of this paper, we use WVS as shorthand for weak virtual synchrony.

The lower bound shown in Lemma 4.1 is valid for weak virtual synchrony as well. However, in this case, there is no need to stop sending messages when the view change protocol is being run. Instead, whenever there is a problem, a suggested view which is a composition of the old view and every process that wishes to join can be introduced to the processes in it. A process that receives a suggested view can send his messages in this suggested view, knowing that some members of the suggested view can be later removed from this view. Finally, when all the messages that were sent in the old view are delivered to all live processes and all the processes that are suspected of being faulty were identified, the "real" new view is introduced to the processes that remains in the "real" view.

\section{Implementing WVS}

In this section we describe the details of a possible implementation of weak virtual synchrony. Our implementation makes the following assumptions:

1. The underlying environment provides reliable (best effort) FIFO communication. That is, if a process $p_{i}$ sends a message to another process $p_{j}$, then the underlying system will do its best to deliver the message, until either $p_{j}$ receives that message, or $p_{i}$ decides to remove $p_{j}$ from its view. Also, every two messages that were sent by the same process to the same process are delivered in the order they were sent.

2. If a process $p_{i}$ stops receiving messages of another process $p_{j}$, either because $p_{i}$ really crashes, because the communication links become too lossy, or because $p_{i}$ has eliminated $p_{j}$ from it's view, then the failure detector of $p_{j}$ will eventually generate a suspect event that includes $p_{i}$. 


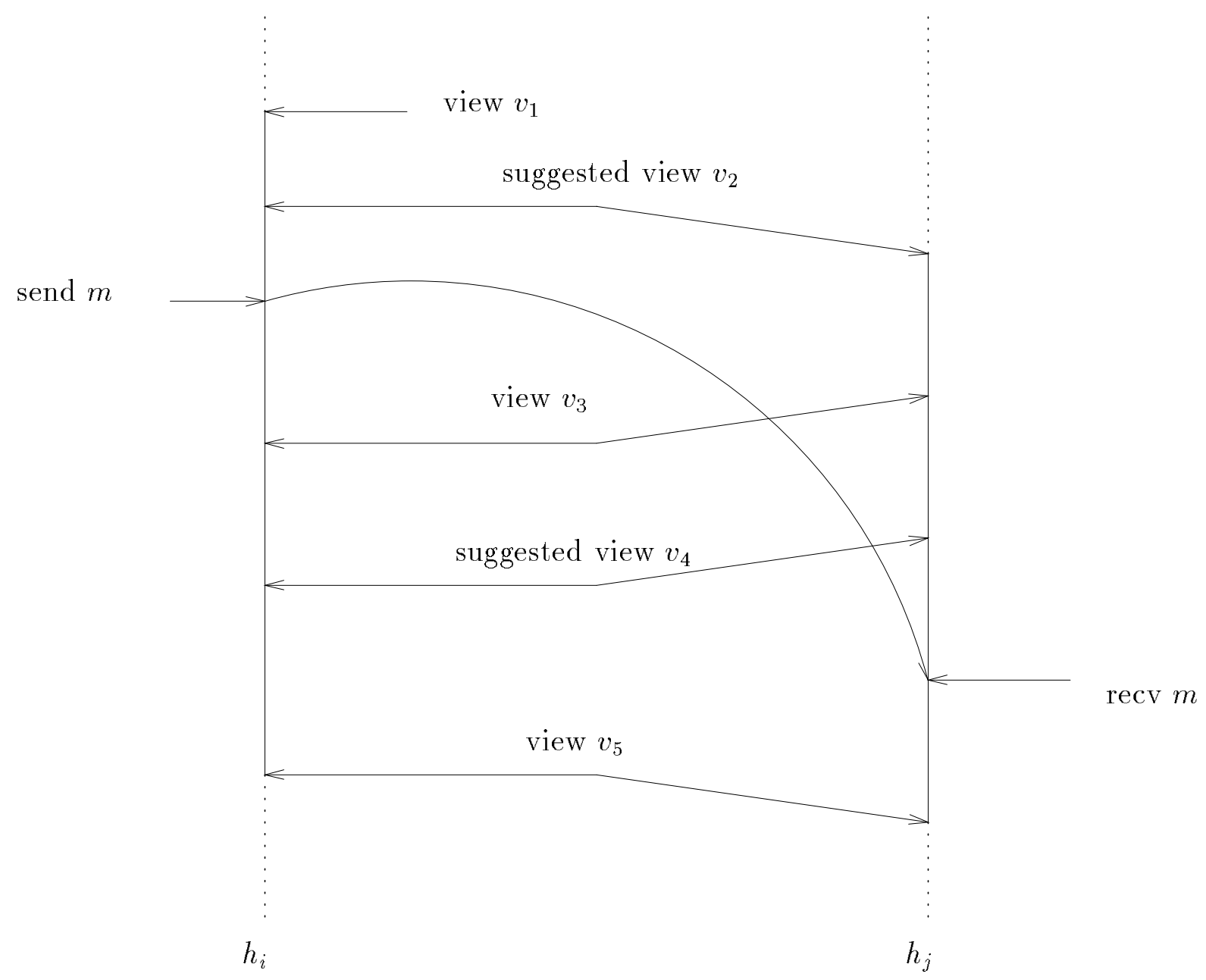

Figure 2: A weakly virtually synchronous execution 
3. If a message becomes stable, i.e., received by every live member of the view, then every live process in the view will eventually learn about it. (We say that a view is stable if all live processes in that view have received it.)

4. Each message is broadcast to all live processes in the suggested view of its invoking process (at the time the message is invoked).

Assumptions 1 and 2 are supported by the NAK layer of Horus, while Assumption 3 is supported by the STABLE layer of Horus. Assumption 4 is a restriction on the layers which run on top of the WVS layer.

Basically, the algorithm goes as follows: Every process initially constructs a view that consists only of itself, and declares itself the contact for that view. Whenever a contact of a view which is already stable learns about another reachable contact with a smaller address, it sends the smaller contact a join request, which includes its view. Note that if the view is not stable, it is not safe to try to join another group; otherwise, some members of the current view may receive the next suggested view before receiving the current view.

A contact that receives a join request, or suspects that a member of its view has failed, and that is not already busy with a view change, starts a view change. This is done by adding to the current view every process that wishes to join (only if this is the first suggested view after a view), and by deleting all processes that are presumed to be faulty. The initiator of a view change also eliminates from the suggested view processes that wish to join, but appear in the current view. This is done to satisfy Condition 5 in the definition of SVS, which must be satisfied by the definition of WVS too: These processes thought that they were separated from the rest of the view and may have refused to receive some of the messages that were received by the rest of the view. Hence, we must eliminate them before allowing them to rejoin. Similarly, whenever a contact has two pending join requests whose views intersect, the contact ignores one of them.

If a process learns about a new suggested view, it adopts this suggested view, and sends all unstable messages from faulty processes to the initiator of the view change. This process then schedules a flushed message to be sent to the new contact immediately after all the messages it has sent in the previous view become stable. Also, if this process was the contact of the previous view, it stops acting as a contact.

If all flushed messages arrive, the initiator of the suggested view adopts the suggested view as the new view and sends all unstable messages that it knows of followed by the new view to all other processes in this view. On the other hand, if before receiving all flushed messages, the initiator of a suggested view receives an indication that a member of this suggested view has failed, then a new suggested view that does not include this faulty member has to be initiated, as described before.

A non-contact process that receives a new view, adopts it. On the other hand, a noncontact process that thinks that all lower ranked processes in its view are faulty, declares itself a contact and initiates a new view change without the processes that it thinks are faulty. 
However, before it sends the newly suggested view to other processes, it must send all unstable messages of the previous contact, so if one of these messages was a view or a suggested view, it will be delivered everywhere before the newly suggested view.

\subsection{Pseudocode}

Each process maintains its own copy of the following variables:

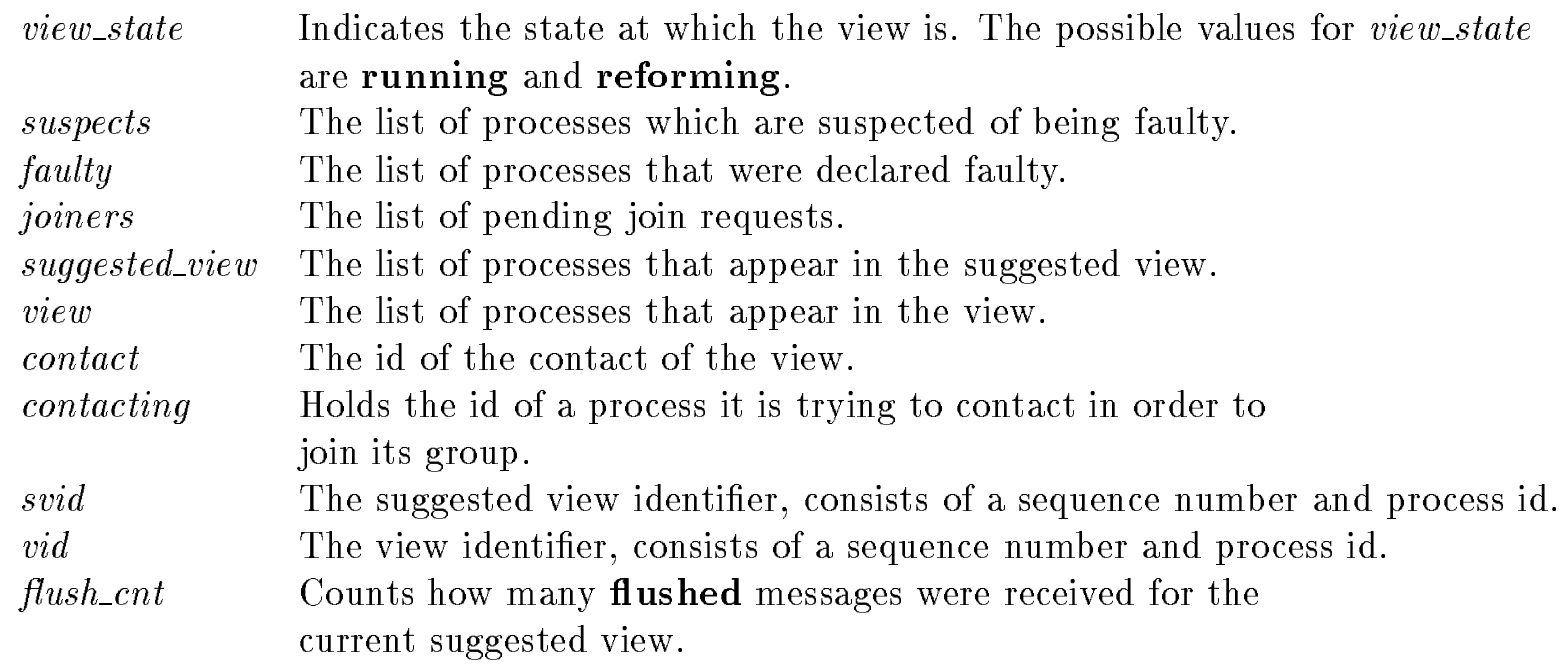

Note that svid and vid can be compared lexicographically, to determine if a certain (suggested) view is "older" or "newer" than another (suggested) view.

When sending and receiving messages, processes use a temporary variable $m s g$, which is a structure that consists of the following fields: type - sview, flushed, join, view, and cast; svid - the value of svid when it was sent; vid - the value of vid when it was sent; contact - the contact of the view; sview - the value of suggested_view when it was sent; view - the value of view when it was sent; faulty - the value of faulty when it was sent. We also make a distinction between the process the initiates a message, and a process that sends the message, since unstable messages can be retransmitted. Processes are not willing to accept messages that are sent from failed members. However, in order to guarantee virtual synchrony, they accept messages that were originated by a faulty member and retransmitted by a live member.

In the code of the algorithm, we denote by - the concatenation of several lists. That is, . preserves the order among members of the original lists and has the property that if one member of list $\mathrm{A}$ appears in the concatenation before a member of list $\mathrm{B}$, then all the members of list $\mathrm{A}$ will appear in the concatenation before all members of list $\mathrm{B}$.

Although most of the code is given as in an event driven form, there are two procedures, init_view_change and create_new_view. These procedures are presented in Figure 3: init_view_change is responsible for generating a new suggested view and distributing it among the members of that suggested view; create_new_view is responsible for adopting a 
suggested view as the next (real) view, and distributing this information among the members of this view. The code for handling net_receive events (messages that are received from the network) is given in Figure 4. In Figure 4 we assume that $p_{i}$ is the process that sent the message. Finally, the code for handling other events is given in Figure 5.

\subsection{Performance Measurements}

In this section we compare the latency of messages sent during view change using both the SVS protocol that is used by Horus and the WVS protocol described in this paper, under various background loads. These measurements were taken on 4 Sparc-20s, connected by a $10 \mathrm{Mbps}$ Ethernet. In order to create the background load, each machine broadcast a message to all other machines in fixed intervals of time. The load on the system is increased by shortening this interval, and is decreased by lengthening this interval. The latency measurements were taken by timestamping each message at each of the layers in Horus, on its way to the Ethernet, and, by using a special device driver, immediately before going out to the Ethernet. At the receiver side, messages were timestamped by our device driver immediately when they were received from the Ethernet, and then at each layer. Using these timestamps, we were able to collect accurate measurements of the one-way latency from the application to the network, denoted by $\delta_{1}$, and the one-way latency from the network to the application, denoted by $\delta_{2}$. If we assume that the time that messages of the same size spend on the wire is the same, then by adding $\delta_{1}$ and $\delta_{2}$, we get a good estimate of the behavior of the real latency.

Since we are running on real systems, there is always the problem of operating system's interference with the measurements. Luckily, all the measurements that we got were either within a factor of three of the average, or at least two order of magnitudes higher than the average. This allowed us to identify measurements which were probably the result of operating systems' related issues, e.g., context switches, and to discard these measurements from the latency graphs.

The results of our measurements appear in Figure 6. As can be seen, the latency of messages sent during view changes in the WVS implementation is higher than the latency of messages sent during normal operation by only a small constant which does not depend on the background load. We explain this difference by the extra overhead associated with handling a suggested view event. On the other hand, messages sent during view changes in the SVS implementation were significantly slower than those sent in the WVS implementation, and the gap between the two implementations increases with the background load.

Also, the results reported in Figure 6 were obtained under fairly simple failure modes, that could have been generated in a systematic manner. Since under more complex failure scenarios it may take much longer to recalculate the new view, we anticipate that in these failure modes the difference between SVS and WVS is even more significant. 


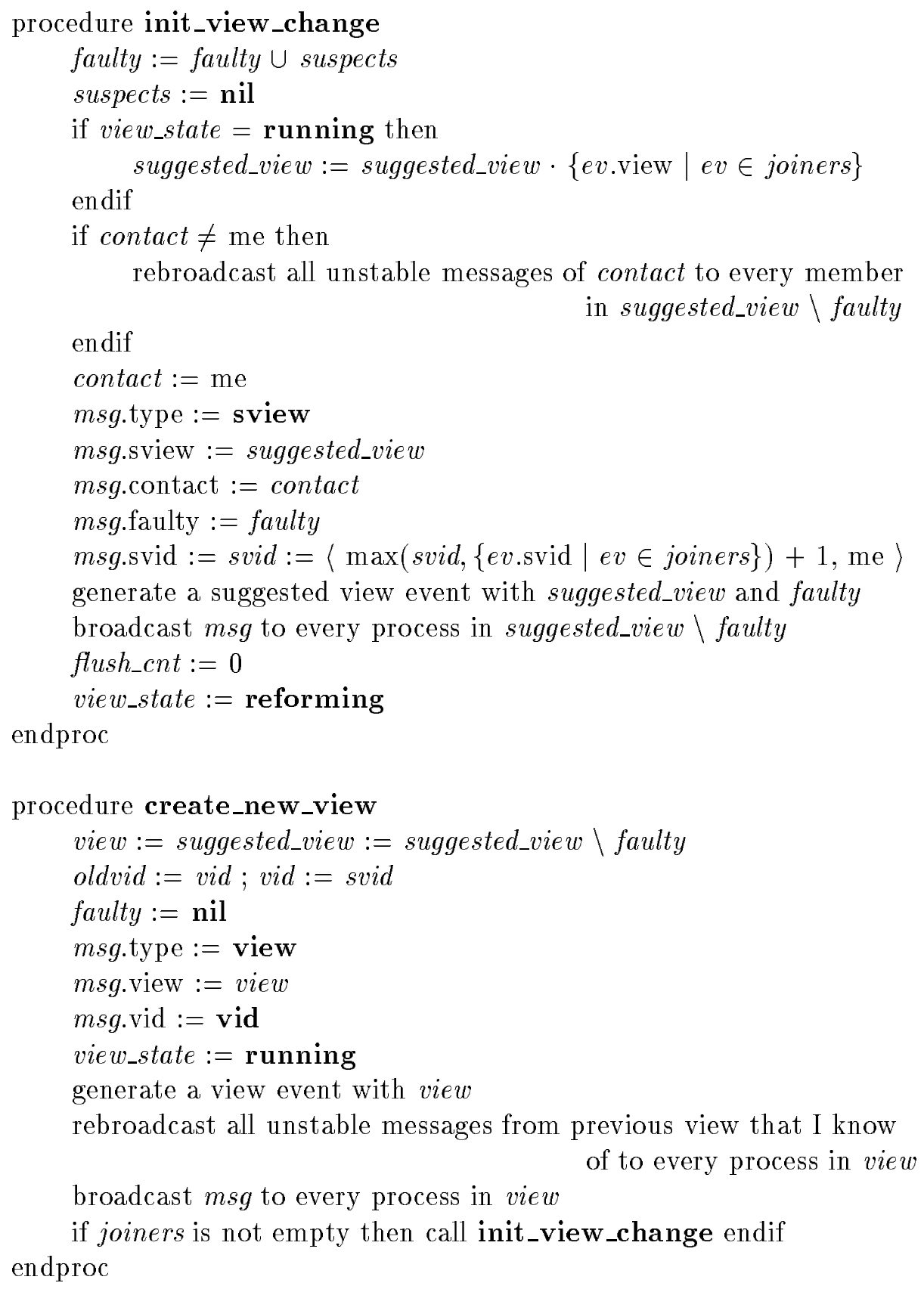

Figure 3: The WVS protocol - procedures 
if msg.type $=$ sview and msg.svid $>$ svid and contacting $=\perp$ then

if vid $\neq$ svid or $m s g$.sview and msg.faulty do not form an ordered subset of

quit suggested_view and faulty then

endif

send every unstable message that was originated by a faulty process to $p_{i}$

view_state $:=$ reforming

suggested_view $:=m s g$. sview

svid $:=$ msg.svid $;$ contact $:=$ msg.contact

faulty $:=$ msg.faulty $;$ suspects $:=$ suspects $\backslash$ faulty

msg.type : = flushed $;$ msg.svid : = svid

schedule to send $m s g$ to $p_{i}$ immediately after all my messages send in the previous

view become stable

generate a suggested view event with suggested_view and faulty

elseif $m s g . t y p e=$ flushed and $m s g$. svid $=$ svid and view_state $=$ reforming then

flush_cnt $:=$ flush_cnt +1

if every process in the suggested view returned a flushed message then

call create_new_view

endif

elseif $m s g . t y p e=$ join and contact and contacting $=\perp$ and $m s g$.view does not overlap with the view of other join requests in joiners then

if $m s g$. view $\cap$ suggested_view $\neq \emptyset$ then

add every process in msg.view $\cap$ suggested_view to suspects

call init_view_change and quit

endif

$e v$. view $:=m s g$.view ; ev.svid $:=m s g$. svid

$e v . p i d:=p_{i}$

joiners $:=$ joiners $\cup$ ev

if view_state $=$ running then call init_view_change endif

elseif $m s g . t y p e=$ view and $m s g . v i d=$ svid and $m s g . v i e w=$ suggested_view $\backslash$ faulty then view $:=$ suggested_view $:=$ msg.view

oldvid $:=$ vid $;$ vid $:=$ msg.vid

faulty $:=$ suspects $:=$ nil

generate a view event with view

elseif $m s g . t y p e=$ cast and $m s g$. svid $>$ oldvid and $p_{i} \in$ suggested_view and

$p_{i} \notin$ faulty $\cup$ suspects then

generate a receive event with $\mathrm{msg}$ endif

Figure 4: The WVS protocol - handling messages (sent by $p_{i}$ ) 
Whenever the failure detector indicate that process $p_{i}$ is faulty do:

if contact then

if contacting $=p_{i}$ then contacting $:=\perp$

else

add $p_{i}$ to suspects

if contacting $=\perp$ then call init_view_change endif endif

elseif all process that appear before me in suggested_view seem to be faulty then add them to suspects and call init_view_change else

add $p_{i}$ to suspects and inform the failure detector of the lowest ranked live process in suggested_view about $p_{i}$

endif

enddo

if contact and contacting $=\perp$ and view_state $=$ running and

the last view is stable and $p_{i}$ is a smaller but reachable contact then

contacting $:=p_{i}$

$m s g . t y p e:=$ join

$m s g$. view $:=$ view

send $m s g$ to $p_{i}$

endif

Whenever the application generates a send event with message $m$ do:

$m s g$. svid $:=$ svid

$m s g . \mathrm{msg}:=m . \mathrm{msg}$

$m s g . t y p e:=$ cast

broadcast $m s g$ to every process in suggested_view $\backslash$ faulty

enddo

Figure 5: The WVS protocol - handling local events 

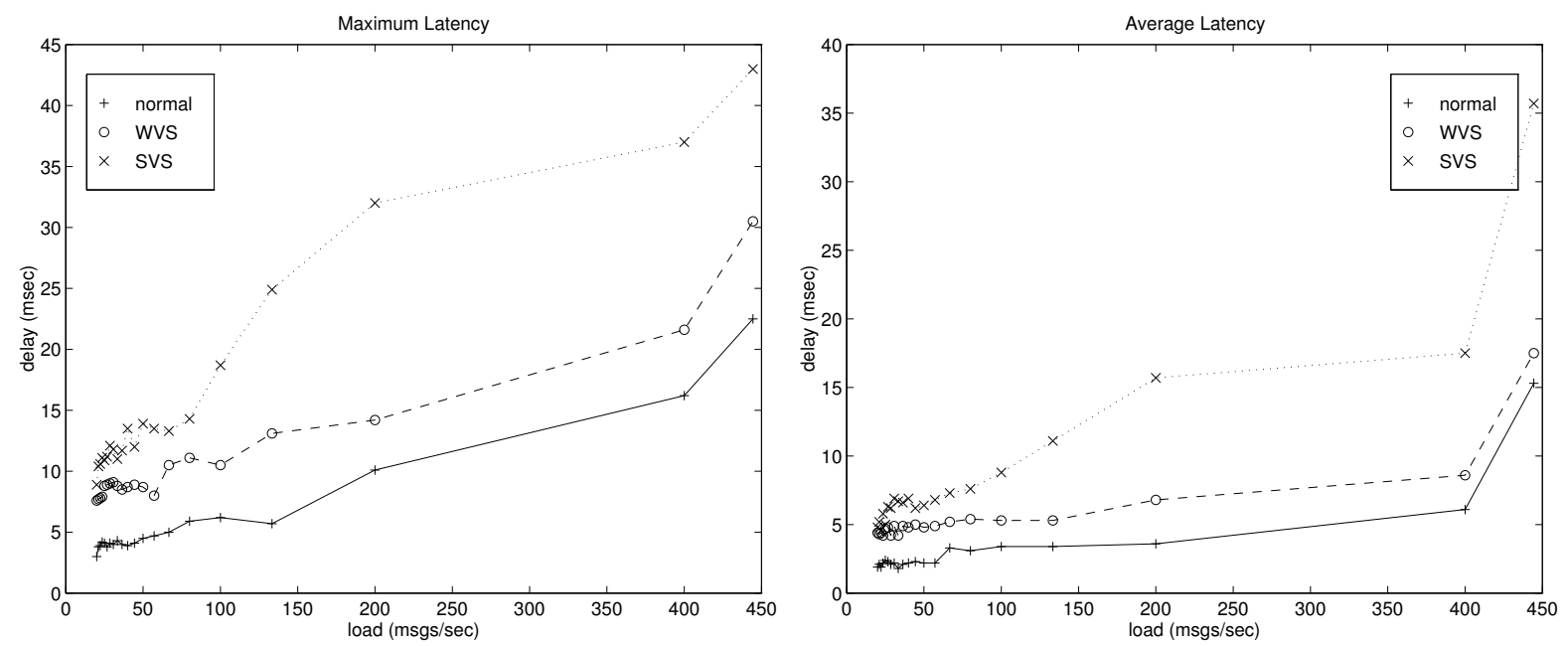

Figure 6: Latency measurements

\subsection{Implementing SVS on top of WVS}

The following lemma states that if the application program would stop sending messages between suggested view events and the following view events, then the resulting semantics would be of strong virtual synchrony.

Lemma 6.1 Let $\sigma$ be a WVS execution in which for every history $h_{i}$ and every send event ev in $h_{i}$, ev is ordered in $h_{i}$ after the view event that generates the view in which ev.msg is sent. Then $\sigma$ is an $S V S$ execution.

If the WVS layer is implemented using the protocol described in Section 6 , then the following scheme can be used to optimize the performance when a process experiences several suggested views before a "real" view is installed: Whenever the application receives a suggested view event, it sends all the messages awaiting to be sent in the "old" view, and only then it allows the membership layer to issue the flush message. This way, messages may never have to be delayed for more than one round of flush messages, although the view change itself may take up to $t+1$ rounds, if $t$ processes crash during this view change.

\section{$7 \quad$ Ordered Virtual Synchrony}

In this section we define ordered virtual synchrony and discuss how Horus' implementation of ordered virtual synchrony can benefit from using WVS instead of SVS.

In order to define ordered virtual synchrony, we must introduce the notion of causal order, originally defined by Lamport in [15]. Given an execution $\sigma$, we say that event ev causally 
precedes event $e v^{\prime}$ if $(a) e v \stackrel{h_{i}}{\longrightarrow} e v^{\prime}$ for some history $h_{i},(b) e v$ is a send event, $e v^{\prime}$ is a receive event and $e v \cdot m s g=e v^{\prime} \cdot m s g$, or $(c)$ there exists another event $e v^{\prime \prime}$ such that $e v$ causally precedes $e v^{\prime \prime}$ and $e v^{\prime \prime}$ causally precedes $e v^{\prime}$.

We assume that send events can be labeled as either abcast, cbcast, or unordered. Given an execution $\sigma$, we define a partial order $\stackrel{\sigma}{\longrightarrow}$ on the receive and send events of $\sigma$ as follows: Let $e v$ and $e v^{\prime}$ be two receive events in $\sigma$ and let $e v^{\prime \prime}$ and $e v^{\prime \prime \prime}$ be the corresponding send events, respectively. Then the following holds:

1. If $e v^{\prime \prime}$ and $e v^{\prime \prime \prime}$ are labeled as abcast, then either $e v \stackrel{\sigma}{\longrightarrow} e v^{\prime}$ or $e v^{\prime} \stackrel{\sigma}{\longrightarrow} e v$. Moreover, if $e v^{\prime \prime} \stackrel{h_{i}}{\longrightarrow} e v^{\prime \prime \prime}$ for some $i$, then $e v \stackrel{\sigma}{\longrightarrow} e v^{\prime}$.

2. If $e v^{\prime \prime}$ is labeled as cbcast, then $e v$ is ordered in $\stackrel{\sigma}{\longrightarrow}$ after any event the causally precedes it in $\sigma$, and before any event that causally follows it in $\sigma$.

We denote by $\stackrel{\sigma}{\longrightarrow} \mid i$ the restriction of $\stackrel{\sigma}{\longrightarrow}$ to events that occur in $h_{i}$. Given a sequence of events $h$ and a partial order $\stackrel{\sigma}{\longrightarrow}$ on the set of events in $h$, we say that $h$ extends $\stackrel{\sigma}{\longrightarrow}$ if for every two events $e v$ and $e v^{\prime}$ in $h$ such that $e v \stackrel{\sigma}{\longrightarrow} e v^{\prime}, e v \stackrel{h}{\longrightarrow} e v^{\prime}$.

Definition 7.1 (Ordered Virtual Synchrony) An SVS (or WVS) execution $\sigma$ is strongly (or weakly) ordered virtually synchronous if there exists a partial order $\stackrel{\sigma}{\longrightarrow}$ such that for every process $p_{i}, h_{i}$ extends $\stackrel{\sigma}{\longrightarrow} \mid i$.

Horus implements ordered virtual synchrony in two layers, called total and causal. We now discuss how these layers of Horus can benefit by having the lower layers implementing WVS instead of SVS.

\subsection{Total Delivery and Causal Delivery}

It is clear that total delivery and causal delivery can be implemented with weaker guarantees than those provided by SVS. However, it is also clear that the total layer cannot deliver a message sent in a new view until all messages of the old view are guaranteed to be delivered. This is due to the possibility that some messages that are missing from the old view were delivered in some of the processes before any of the messages of the new view. However, we believe that a protocol for implementing total delivery can still benefit from WVS since even in cases as described above, messages are delayed at the receiver side and not at the sender side. Hence, when the "real view" is installed, the delivery of these messages does not require any network traffic.

As for the causal layer, in the worst case, a message may have to wait for the new view to be installed, like in the total layer, before it can be delivered. However, from our experience, messages of a new view can often be delivered before the "real" view is installed, since usually none of the missing messages from previous views causally precede them. In particular, local deliveries do not have to be blocked at all. 


\section{Multiple Groups}

Up until now, the discussion about virtual synchrony was limited to the case were there is only one group. However, in practice, there can be many groups and every process may be a member of several groups simultaneously. This, of course, can be modeled in our framework by adding a new field called group to every event and then use the definitions of Sections 4 and 5 on the restriction of executions and/or histories to events of the same group. For example, an execution $\sigma$ is SVS (WVS) if for every group $g, \sigma \mid g$, the restriction of $\sigma$ to events of group $g$, is an SVS (WVS) execution according to the definitions of Section 4 (5).

Although the above definition correctly describes the guarantees provided by Horus, Horus provides an additional guarantee known as failure domains. That is, if a process is a member of several groups and is declared or suspected of being faulty, then it is declared or suspected of being faulty in all the groups it belongs to. The following is a suggested definition for failure domains:

Definition 8.1 (Failure Domain) Given a set of groups $G$, we say that $G$ shares the same failure domain if the following holds: For every history $h_{j}$ and every process $p_{i}$, if $h_{j}$ includes a suspect event ev such that $p_{i} \in$ ev.suspects, then for every group $g \in G$ for which $h_{j}$ includes a view event $e v^{\prime}$ such that $e v^{\prime} . g r o u p=g$ and $p_{i} \in e v^{\prime} . v i e w$, there exists another event $e v^{\prime \prime}$ such that $e v^{\prime} \stackrel{h_{j}}{\longrightarrow} e v^{\prime \prime}, e v^{\prime \prime} . g r o u p=g$, and either $e v^{\prime \prime}$ is a suspect event such that $p_{i} \in e v^{\prime \prime}$. suspects or $e v^{\prime \prime}$ is a view event such that $p_{i} \notin e v^{\prime \prime}$.view.

This definition is slightly weaker than the behavior of failure domains when a process is suspected of being faulty more than once. Unfortunately, we are currently not aware of any simple way to define this behavior formally and precisely.

\section{Discussion}

Our current research with Horus is aimed at developing a tool that will provide the application with a semantics which is strong enough to cope with failures, while ensuring low message latencies, even in "bad" scenarios. In this paper we take a step towards this goal, by looking at the membership layer of Horus. We presented a formal definition of strong virtual synchrony, capturing the guarantees provided by this layer. Using this formal definition, we were able to identify sources for long delays in this layer, and to suggest a way to overcome these delays without sacrificing the desired properties of the existing semantics, namely, by introducing the notion of weak virtual synchrony. We have developed an implementation for weak virtual synchrony and showed that indeed, in our system, weak virtual synchrony does not incur the same long latencies as strong virtual synchrony.

In addition to the membership layer, Horus has several other layers that may incur unbounded latencies. Hence, we would like to study these layers too, and make sure that, at 
least with very high probability, we can eliminate the sources of uncertainty from these layers as well. At the moment we are also studying the layer that is responsible for providing total delivery of messages, and are looking at the behavior of several total delivery protocols.

In order to reach a higher degree of confidence in our protocols, we would like to formally prove their correctness. This can be either by composing formal proofs of correctness, or by using automatic verification tools. For this, the formal definitions presented in this papers will be very helpful.

We believe that it is possible to slightly modify the protocol developed in Section 6 to make it self stabilizing [11] under certain assumptions. For example, if we assume that processes have a unique name, that cannot be corrupted, then we believe that by adding several sanity checks, this protocol would become self stabilizing. If the processes' name can be corrupted, but they have a way to retrieve it, e.g, the name of a process is the address of the network device and this address cannot be corrupted, then we believe that by verifying the name every so often, and by adding some sanity checks, the protocol would also become self stabilizing.

Acknowledgements: We would like to thank Ken Birman, David Karr, and Werner Vogels for many helpful discussions and comments. We would also like to thank Gil Neiger and Özalp Babaoğlu. 


\section{References}

[1] Y. Amir, D. Dolev, S. Kramer, and D. Malki. Transis: A Communication Sub-System for High Availability. In Proc. of the 22nd Annual International Symposium on Fault-Tolerant Computing, pages 76-84, July 1992.

[2] Y. Amir, D. Dolev, S. Kramer, and D. Malki. Membership Algorithms in Broadcast Domains. In Proc. of the 6th International Workshop on Distributed Algorithms, Lecture Note in Computer Science \#647, pages 292-312, November 92.

[3] Y. Amir, L. E. Moser, P. M. Melliar-Smith, D.A. Agarwal, and P. Ciarfella. Fast Message Ordering and Membership Using a Logical Token-Passing Ring. In Proc. of the 13th International Conference on Distributed Computing Systems, pages 551-560, May 1993.

[4] Ö. Babaoğlu, R. Davoli, L. Giachini, and M. Baker. Relacs: A Communication Infrastructure for Constructing Reliable Applications in Large-Scale Distributed Systems. Technical Report UBLCS-94-15, Department of Computer Science, University of Bologna, June 1994. Revised January 1995.

[5] K. Birman. The Process Group Approach to Reliable Distributed Computing. Communications of the ACM, 36(12):37-53, December 1993.

[6] K. Birman. Integrating Runtime Consistency Models for Distributed Systems. Journal of Parallel and Distributed Computing, 23:158-176, 1994.

[7] K. Birman and T. Joseph. Exploiting Virtual Synchrony in Distributed Systems. In Proc. of the 11th ACM Symp. on Operating Systems Principles, pages 123-138, December 1987.

[8] K. Birman and T. Joseph. Reliable Communication in the Presence of Failures. ACM Transactions on Computer Systems, 5(1):47-76, February 1987.

[9] K. Birman, A. Schiper, and P. Stephenson. Lightweight Causal and Atomic Group Multicast. ACM Transactions on Computer Systems, 9(3):272-314, August 1991.

[10] J. Chang and N. Maxemchuk. Reliable Broadcast Protocols. ACM Transactions on Computer Systems, 2(3):251-273, August 1984.

[11] E. W. Dijkstra. Self-stabilizing Systems in Spite of Distributed Control. Communications of the $A C M, 17(11): 643-644$, November 1974.

[12] P. Ezhilchelvan, R. Macedo, and S. Shrivastava. Newtop: A Fault-Tolerant Group Communication Protocol. Technical report, Computer Science Department, University of Newcastle, Newcastle upon Tyne, United Kingdom, August 1994.

[13] A. Gopal and S. Toueg. Reliable Broadcast in Synchronous and Asynchronous Environments. In J.-C. Bermond and M. Raynal, editors, Proceeding of the Third International Workshop on Distributed Algorithms, volume 392 of Lecture Notes on Computer Science, pages 110-123. Springer-Verlag, September 1989. 
[14] R. Guerraoui. Transaction model vs Virtual Synchrony model: bridging the gap. In K. Birman, F. Cristian, F. Mattern, and A. Schiper, editors, Distributed Systems: From Theory to Practice, Lecture Notes on Computer Science. Springer-Verlag, 1995. To appear.

[15] L. Lamport. How to Make a Multiprocessor Computer that Correctly Executes Multiprocess Programs. IEEE Trans. on Computers, C-28(9):690-691, 1979.

[16] B. Liskov, S. Ghemawat, R. Gruber, P. Johnson, L. Shrira, and M. Williams. Replication in the Harp File System. In Proceeding of the 13th ACM Symposium on Operating Systems Principles, pages 226-238, Pacific Grove, CA, October 1991.

[17] S. Maffeis, W. Bischofberger, and K. Matzel. A Generic Multicast Transport Service to Support Disconnected Operation. In Proceeding of the 2nd USENIX Symposium on Mobile and Location-Independent Computing, pages 79-89, Ann Arbor, MI, April 1995.

[18] M. Makpangou and K. Birman. Designing Application Software in Wide Area Network Settings. Technical Report 90-1165, Departmenr of Computer Science, Cornell University, October 1990.

[19] S. Mishra, L. Peterson, and R. Schlichting. Consul: A Communication Substrate for Fault-Tolerant Distributed Programs. Distributed Systems Engineering Journal, 1(2):87103, December 1993.

[20] G. Neiger and S. Toueg. Automatically Increasing the Fault-Tolerance of Distributed Algorithms. Journal of Algorithms, 11(3):374-419, September 1990.

[21] L. Peterson, N. Buchholz, and R. D. Schlichting. Preserving and Using Context Information in Interprocess Communication. ACM Transactions on Computer Systems, $7(3): 217-246$, August 1989.

[22] A. Ricciardi and K. P. Birman. Consistent Process Membership in Asynchronous Environments. In K. Birman and R. van Renesse, editors, Reliable Distrinuted Computing With The ISIS Toolkit, chapter 13. IEEE Computer Society Press, Los Alamitos, 1993.

[23] A. Schiper and A. Sandoz. Uniform Reliable Multicast in a Virtually Synchronous Environent. In Proc. of the 13th International Conference on Distributed Computing Systems, pages 561-568, May 1993.

[24] R. van Renesse, K. Birman, R. Friedman, M. Hayden, and D. Karr. A Framework for Protocol Composition in Horus. In Proc. of the 14th ACM Symposium on Principles of Distributed Computing, pages 80-89, August 1995.

[25] R. van Renesse, K. P. Birman, and T. M. Hickey. Design and Performance of Horus: A Lightweight Group Communications System. Technical Report 94-1442, Cornell University, Dept. of Computer Science, August 1994.

[26] R. van Renesse, R. Friedman, and D. Malki. Understanding Virtual Synchrony. In preparation. 